\title{
Percutaneous balloon pericardiotomy in a child with recurrent pericardial effusions due to systemic lupus erythematosus
}

\author{
Sunethra Irugal Bandara ${ }^{1}$, WA D W Karunarathne ${ }^{2}$, Evone Jayaweera ${ }^{2}$, G I M Jinadasa ${ }^{2}$
}

Sri Lanka Journal of Child Health, 2013; 42(3):

(Key words: Percutaneous balloon pericardiotomy; recurrent pericardial effusion)

\section{Introduction}

Recurrent symptomatic pericardial effusions can complicate different cardiac and extracardiac diseases. When recurrent pericardial effusions occurred, after drainage with conventional catheter techniques, the creation of a pericardial window by open surgery used to be the unique treatment available until the recent development of percutaneous balloon pericardiotomy (PBP). In this report we describe the creation of a pericardial window by static balloon dilatation in a patient who had recurrent pericardial effusion with tamponade due to systemic lupus erythematosus (SLE), a rare occurrence $^{1}$. This is the only reported case of PBP in Sri Lanka.

\section{Case report}

A 12 year old girl was transferred from General Hospital, Nuwara Eliya with chest pain and shortness of breath of 2 weeks duration for echocardiography. On examination, the pulse rate was 110 per minute and the pulse was of low volume. Blood pressure (BP) was $82 / 52 \mathrm{mmHg}$. Jugular venous pressure (JVP) was raised with a respiratory rate of 32 per minute. Echocardiography revealed a large pericardial effusion all around the heart with right ventricular collapse. Pericardiocentesis was done and a pigtail catheter was left in the pericardial cavity. Fluid was sent for investigations.

The pigtail catheter was kept for seven days with echocardiography and aspiration of $180-260 \mathrm{ml}$ of

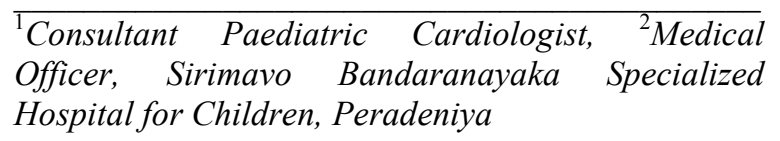

(Received on 28 September 2012: Accepted after revision on 9 November 2012) fluid almost daily. The catheter was removed on the seventh day. She developed cardiac tamponade on the ninth day. Repeat pericardiocentesis was done and the catheter was kept in situ. She was managed in the intensive care unit because of her poor general condition and 14 pericardial aspirations were done over 3 weeks. Therefore, it was decided to perform PBP.

Patient was taken into the cardiac catheterization laboratory and the procedure was performed under local anaesthesia. Prophylactic antibiotics were administered before the procedure. The procedure was performed under fluoroscopic and echocardiographic guidance. Percutaneous subxiphoid approach was used to insert a pericardial catheter for pericardiocentesis. Catheter was removed and short sheaths up to $7 \mathrm{~F}$ were inserted. $5 \mathrm{~F}$ pigtail catheter was passed over 0.035 guide wire into the pericardial space. $50 \mathrm{ml}$ of $25 \%$ diluted contrast medium was injected into the pericardial cavity to identify the parietal pericardium. The pericardial catheter was removed and 0.035 guide wire was placed in the pericardial cavity. A $16 \mathrm{~mm}$ diameter, 3cm-long balloon dilating catheter (Tyshak 11 balloon) was advanced over the guide wire.

The balloon was partially inflated with a mixture of equal volumes of contrast medium and saline and gradually withdrawn until there was a clear waist on the middle portion of the balloon due to the tight pericardium. The balloon was then inflated manually until the waist disappeared (Figure 1). Three inflations for 2-3 minutes were performed to assure an adequate opening of the parietal pericardium. After the dilation, the balloon was removed and a $5 \mathrm{~F}$ pigtail catheter was inserted into the pericardial space. The catheter was removed after 24 hours with echocardiographic check. 

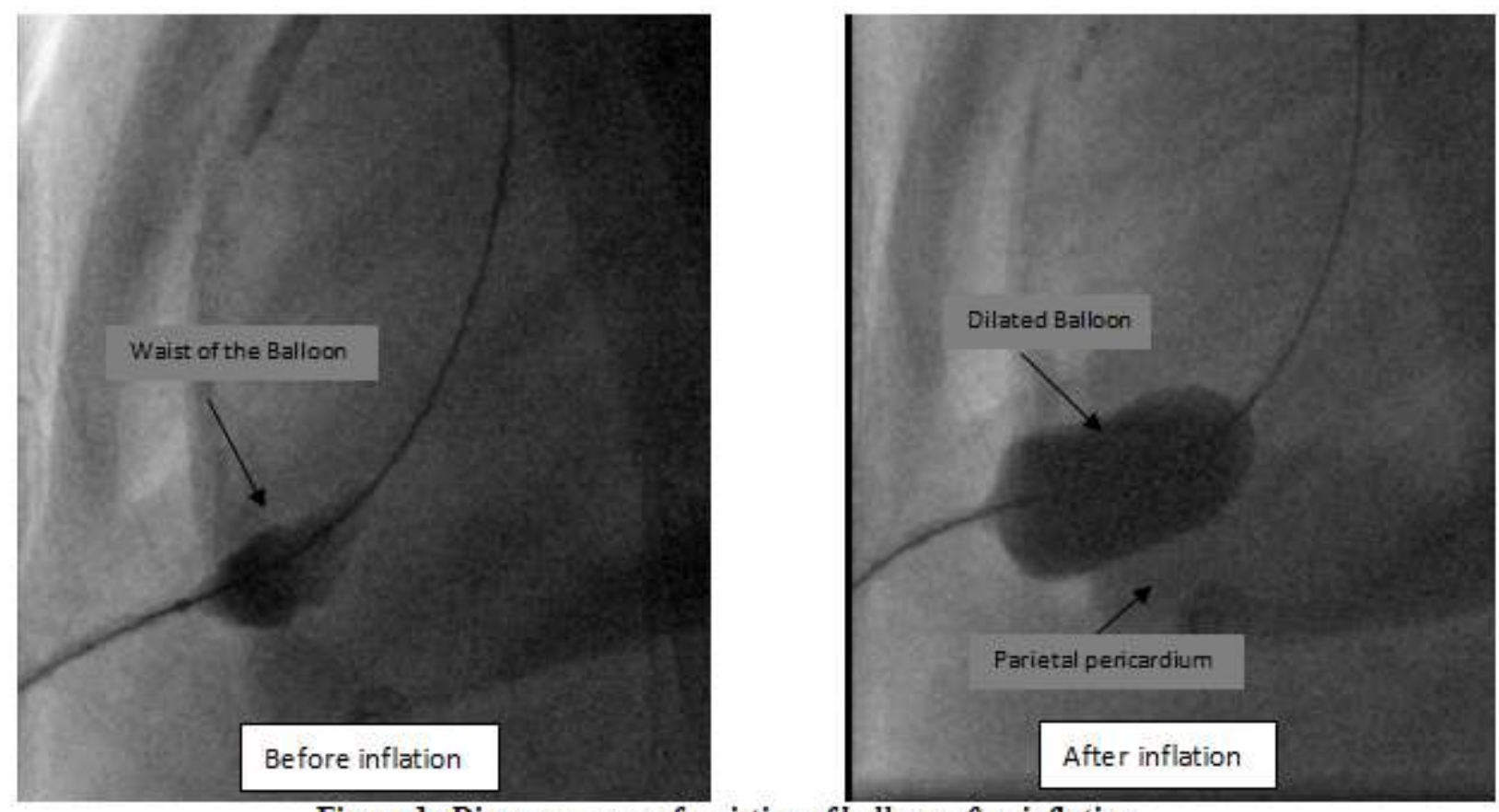

Figure 1: Disappearance of waisting of balloon after inflation

Chest radiographs were taken before, immediately after and 24 hours after the procedure to exclude pneumothorax and to monitor the development of a left pleural effusion. Follow up echocardiograms did not reveal significant pericardial effusion even after 4 months following the procedure.

\section{Discussion}

PBP was initially introduced by Dr. Palacios in USA in 1991 and was mainly indicated for recurrent malignant pericardial effusion when surgery was too risky, since surgical pericardial window creation in critically ill patients requires general anaesthesia and carries an increased risk of morbidity and mortality ${ }^{2}$. PBP has been performed mainly in patients with malignant effusions as well as in miscellaneous cases of pericardial effusion with tamponade, recurrent pericardial effusions, or initial therapy at the time of pericardiocentesis.

The most common causes of pericardial effusion identified are infections, cardiac surgery and malignancy. It can also occur after trauma, hypersensitivity and autoimmune related-systemic lupus erythematosus, rheumatic fever and rheumatoid arthritis, drug therapy, uraemia and idiopathic pericardial effusion ${ }^{1,3}$.

Patient presentation is either asymptomatic or with symptoms of cardiac tamponade. Usually pericardial disease due to SLE is not haemodynamically significant. Occasionally, the effusion can be very large. Tamponade is rare and development of constrictive pericarditis is rarer still. Commonly, a mild pleuritis accompanies the pericarditis ${ }^{2}$. In this patient SLE was confirmed and there was a significant left sided pleural effusion which was drained.

In cardiac tamponade, initial treatment is pericardiocentesis followed by surgical creation of pericardial window or PBP which is a less invasive approach as compared to surgery if there is repeated accumulation of pericardial effusion. Immediate management of tamponade relieves the acute symptoms, while long term prognosis depends on the cause of the effusion, regardless of the intervening procedure $^{4,5}$.

Our patient who had SLE with poor general condition needing intensive care was a poor candidate for surgical intervention. PBP technique is relatively simple and safe. Balloon inflation causes localized tearing of parietal pericardial tissue leading to communication of pericardial space with pleural space or with abdominal cavity.

The complications of the procedure are pleural effusion, pneumothorax, atrial or ventricular puncture, subcutaneous emphysema and infection. Finally, risks of the procedure in experienced hands do not exceed pericardiocentesis risk performed by most cardiologists; however, if done by non- trained 
hands, this could lead easily to rupture of the myocardium.

Percutaneous balloon pericardiotomy is an easy and useful technique to manage patients with large recurrent pericardial effusions with a low rate of complications.

\section{References}

1. Allen HD, Driscoll DJ, Shaddy RE, Feltes TF. Moss and Adams' Heart Disease in Infants, Children and Adolescents, $7^{\text {th }}$ ed. vol 1, 1286-90

2. Mills SA, Julian S, Holliday RH. Subxiphoid pericardial window for pericardial effusive disease. Journal of Cardiovascular Surgery 1989; 30:768-73.
3. Strimel WJ, Fredi JL. Pericardial effusion. Available from:

http://emedicine.medscape.com/article/157325overview (Updated July 13, 2012)

4. Ziskind AA, Pearce AC, Lemmon $\mathrm{CC}$ et al. Percutaneous balloon pericardiotomy for the treatment of cardiac tamponade and large pericardial effusions: Description of technique and report of the first 50cases. Journal of American College of Cardiology 1993;21:1-5. http://dx.doi.org/10.1016/0735-1097(93)90710-I

5. Puri A, Aquarwal N, Dwiwedi SK, Narain VS. Percutaneous balloon pericardiotomy for treatment of recurrent malignant pericardial effusion. Indian Heart Journal 2012; 64(1): 8889.

http://dx.doi.org/10.1016/S0019-4832(12)60018-2 